ARTICLE

\title{
Severe T cell hyporeactivity in ventilated COVID-19 patients correlates with prolonged virus persistence and poor outcomes
}

Kerstin Renner ${ }^{1}$, Tobias Schwittay ${ }^{1}$, Sophia Chaabane${ }^{1}$, Johanna Gottschling ${ }^{1}$, Christine Müller ${ }^{1}$, Charlotte Tiefenböck', Jan-Niklas Salewski , Frederike Winter ${ }^{1,2}$, Simone Buchtler ${ }^{1}$, Saidou Balam (1) 1, Maximilian V. Malfertheiner (10 ${ }^{3}$, Matthias Lubnow ${ }^{3}$, Dirk Lunz ${ }^{4}$, Bernhard Graf ${ }^{4}$, Florian Hitzenbichler ${ }^{5}$, Frank Hanses ${ }^{5}$, Hendrik Poeck ${ }^{6}$, Marina Kreutz ${ }^{6}$, Evelyn Orsó ${ }^{7}$, Ralph Burkhardt (1) 7 , Tanja Niedermair ${ }^{8,9}$, Christoph Brochhausen ${ }^{8,9}$, André Gessner ${ }^{10}$, Bernd Salzberger ${ }^{5}$ \& Matthias Mack (1) ${ }^{1,2 凶}$

Coronavirus disease 2019 (COVID-19) can lead to pneumonia and hyperinflammation. Here we show a sensitive method to measure polyclonal $\mathrm{T}$ cell activation by downstream effects on responder cells like basophils, plasmacytoid dendritic cells, monocytes and neutrophils in whole blood. We report a clear T cell hyporeactivity in hospitalized COVID-19 patients that is pronounced in ventilated patients, associated with prolonged virus persistence and reversible with clinical recovery. COVID-19-induced T cell hyporeactivity is T cell extrinsic and caused by plasma components, independent of occasional immunosuppressive medication of the patients. Monocytes respond stronger in males than females and IL-2 partially restores T cell activation. Downstream markers of $\mathrm{T}$ cell hyporeactivity are also visible in fresh blood samples of ventilated patients. Based on our data we developed a score to predict fatal outcomes and identify patients that may benefit from strategies to overcome $T$ cell hyporeactivity.

\footnotetext{
${ }^{1}$ Department of Nephrology, University Hospital Regensburg, Regensburg, Germany. ${ }^{2}$ Regensburg Center for Interventional Immunology, Regensburg, Germany. ${ }^{3}$ Department of Internal Medicine II, University Hospital Regensburg, Regensburg, Germany. ${ }^{4}$ Department of Anesthesiology, University Hospital Regensburg, Regensburg, Germany. ${ }^{5}$ Department of Infection Prevention and Infectious Diseases, University Hospital Regensburg, Regensburg, Germany. ${ }^{6}$ Department of Internal Medicine III, University Hospital Regensburg, Regensburg, Germany. ${ }^{7}$ Institute of Clinical Chemistry and Laboratory Medicine, University Hospital Regensburg, Regensburg, Germany. ${ }^{8}$ Institute of Pathology, University of Regensburg, Regensburg, Germany. ${ }^{9}$ Central Biobank Regensburg, University and University Hospital, Regensburg, Germany. ${ }^{10}$ Institute Clinical Microbiology and Hygiene, University Hospital

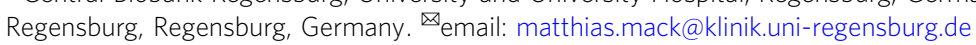


S ARS-CoV-2, the etiologic agent of coronavirus disease 19 (COVID-19), was discovered in Wuhan (China) in December 2019 and became pandemic within a couple of months ${ }^{1}$. Most patients with SARS-CoV-2 infection are asymptomatic or show only mild symptoms, while some patients develop bilateral interstitial pneumonia and require oxygen support by nasal cannula or mechanical ventilation on an intensive care unit $(\mathrm{ICU})^{2}$.

Several risk factors for COVID-19 associated death have been identified including age, gender, adipositas, pulmonary diseases, and diabetes ${ }^{3-6}$. Acute respiratory distress syndrome (ARDS) and fatal outcomes are also associated with hyperinflammation and microthrombosis ${ }^{7}$. Activated monocytes/macrophages contribute to death in a mouse model of SARS-CoV infection ${ }^{8}$ and also seem to play an important role in hyperinflammation in human SARSCoV-2 infection 9,10 . Monocytes are activated by a wide range of signals, including pathogen-derived signals (e.g. nucleic acids) and T-cell-derived signals (e.g. cytokines) ${ }^{11}$. The innate immune system seems to be insufficient to rapidly clear SARS-CoV-2 infection by itself, as SARS-CoV-2-specific T- and B-cell responses develop in most patients ${ }^{12-14}$. Virus-specific T-cell responses and neutralizing antibodies, typically generated in a $\mathrm{T}$ cell-dependent manner, are considered essential for viral clearance ${ }^{15}$. Potential benefits of plasma obtained from convalescent COVID-19 patients also points into this direction ${ }^{16,17}$. On the other hand, hyperinflammation in COVID-19 patients might be caused by excessive T-cell responses that activate monocytes and neutrophils. Beneficial effects of dexamethasone indicate that hyperinflammation contributes to fatal outcomes $^{18,19}$. However, dexamethasone does not only interfere with T-cell activation and could also exert therapeutic effects by blocking excessive innate immune responses against viral components.

Current data on polyclonal T-cell reactivity in COVID-19 are conflicting and indicate that patients with severe COVID-19 can have either insufficient ${ }^{20,21}$ or excessive $\mathrm{e}^{22-24} \mathrm{~T}$-cell responses, as recently summarized ${ }^{25}$. T-cell responses were measured by intracellular cytokine staining of PBMCs, cultured with phorbolesters and ionomycin, by quantification of cytokines after polyclonal stimulation, or by expression of surface markers on T cells. IFN-gamma expression for example was found to be unchanged $^{26}$, increased ${ }^{23,24}$, or decreased ${ }^{20,21}$. In contrast, SARSCoV-2-specific T-cell responses were repeatedly reported to be reduced in critically ill and older COVID-19 patients and associated with an unfavorable outcome o $^{27,28}$.

We applied a sensitive method to analyze polyclonal T-cell reactivity in COVID-19 patients and controls. T-cell activation was measured by downstream effects on responder cells, such as basophils, pDCs, monocytes, and neutrophils in whole blood. These assays turned out to be much more sensitive and consistent than classical readouts with PBMCs. We found that impaired Tcell reactivity is already evident in mild disease, caused by plasma components present in COVID-19 patients and, strongly associated with disease severity and prolonged viral replication. Gender-specific differences in T-cell-induced downstream effects on monocytes were observed. Finally, we developed a score to predict the fatal outcome to identify patients that may benefit from strategies to overcome T-cell hyporeactivity.

\section{Results}

Clinical characterization of COVID-19 patients. We analyzed 55 patients with COVID-19 infection admitted to the University Hospital Regensburg from March to July 2020. SARS-CoV-2infection was diagnosed by RT-PCR from oropharyngeal swabs. Patients were stratified into "non-ventilated" and "ventilated" patients according to the need for mechanical ventilation. Ventilated patients were further subgrouped into "survived", if they could be discharged from the ICU, or into "dead" if they died in the ICU. In addition, 42 healthy controls were included in the study. Clinical characteristics of patients and controls are shown in Table 1. In most patients, several consecutive blood samples were available resulting in a total of 188 samples. In total, 14 patients were first sampled on mechanical ventilation and also after weaning from ventilation. Samples of these patients were assigned into the subgroup "ventilated" or "non-ventilated" according to the ventilation status at the time of sampling (Supplementary Table 1).

In accordance with published data ${ }^{3,4}$, male gender was more frequent in ventilated than non-ventilated patients (80 versus $52 \%)$. Ventilated patients also had a highly significant longer period of virus replication (28 days versus 10 days) that was defined as the period from the day of first clinical symptoms to the day of the last positive virus RT-PCR. Comorbidities were similar in both groups, while bacterial superinfection, thromboembolic complications, and treatment with antibiotics or convalescent plasma were more frequent in ventilated patients (Table 1). As described before ${ }^{29}$, ventilated patients displayed higher plasma concentrations for biomarkers indicating inflammation (CRP, IL-6), liver and muscle dysfunction (LDH, Bilirubin, CK), and intravascular coagulation (D-dimer). Ventilated "dead" patients showed higher leukocyte counts and higher biomarkers for inflammation and liver dysfunction than ventilated "survived" patients (Supplementary Table 1).

Impaired polyclonal T-cell reactivity is a hallmark of COVID19 patients and correlates with disease severity. To measure Tcell reactivity, we used fresh heparinized whole blood and polyclonally activated the $\mathrm{T}$ cells with immobilized anti-CD3 antibodies. T-cell activation was quantified not only by cytokine release but also by downstream effects on various cell types including basophils, pDCs, monocytes, and neutrophils. In preparation for the study, we have established that T-cell activation results in strong activation of basophils measured by upregulation of CD203c and downregulation of the IL-3-receptor beta-chain CD131 (used in this study). Basophils strongly express IL-3receptors but only low levels of GM-CSF- and IL-5-receptors that also use CD131 for signal transduction ${ }^{30}$. Thus, among a variety of cytokines IL-3 is mainly responsible for downregulation of CD131 on basophils (Supplementary Fig. 1a). pDCs express IL-3and GM-CSF-receptors; thus both cytokines contribute to downregulation of CD131 on pDCs (Supplementary Fig. 1b). $\mathrm{CD} 14+$ monocytes respond to T-cell activation by a very pronounced upregulation of the IL-3-receptor alpha-chain CD123. Various cytokines, including IL-3, GM-CSF, IL-2, IL-15, IFNgamma, IFN-alpha, TNF-alpha induce a strong upregulation of CD123 on monocytes (Supplementary Fig. 1c). Thus monocytes integrate a larger set of direct and indirect T-cell-derived signals. Similarly, neutrophils respond to a large set of cytokines with upregulation of CD11b (Supplementary Fig. 1d).

T-cell activation with immobilized anti-CD3 in whole blood of healthy controls resulted in a pronounced downregulation of CD131 on basophils (to 13\%) (Fig. 1a) as well as upregulation of CD123 on monocytes (to 1225\%) (Fig. 1c) and CD11b on neutrophils (to 1232\%) (Fig. 1e). In COVID-19 patients these changes were much weaker. Ventilated COVID-19 patients showed no significant downregulation of CD131 on pDCs, a weak downregulation of CD131 on basophils (to 77\%), a weak upregulation of CD123 on monocytes (to 345\%), and of CD11b on neutrophils (to 424\%). Responses of non-ventilated patients were in-between the responses of ventilated patients and healthy 
Table 1 Demographics and clinical information from study patients and healthy controls.

\begin{tabular}{|c|c|c|c|c|c|c|}
\hline & Healthy & COVID-19 all & $\begin{array}{l}\text { COVID-19 non- } \\
\text { ventilated }\end{array}$ & $\begin{array}{l}\text { COVID-19 } \\
\text { ventilated all }\end{array}$ & $\begin{array}{l}\text { COVID-19 ventilated } \\
\text { survived }\end{array}$ & $\begin{array}{l}\text { COVID-19 } \\
\text { ventilated dead }\end{array}$ \\
\hline Number of patients $(n)$ & 42 & 55 & 25 & 30 & 23 & 7 \\
\hline \multicolumn{7}{|l|}{ Demographics } \\
\hline Mean age (years, min-max) & $37.8^{a}(19-78)$ & $56.8(19-78)$ & $55.1(19-78)$ & $58.3(31-73)$ & $57.7(31-73)$ & $60.1(51-68)$ \\
\hline Sex $(\%$ male $)$ & $15(36 \%)^{b}$ & $37(67 \%)$ & $13(52 \%)$ & $24(80 \%)^{c}$ & $17(74 \%)$ & $7(100 \%)$ \\
\hline \multicolumn{7}{|l|}{ Virus persistence } \\
\hline Mean, days & & 20 & 10 & $28^{d}$ & 29 & 23 \\
\hline \multicolumn{7}{|l|}{ Comorbidities, $n(\%)$} \\
\hline Smoking & & $5(9 \%)$ & $4(16 \%)$ & $1(3 \%)$ & $1(4 \%)$ & 0 \\
\hline Hypertension & & $28(51 \%)$ & $11(44 \%)$ & $17(57 \%)$ & $12(52 \%)$ & $5(71 \%)$ \\
\hline Diabetes mellitus & & $15(27 \%)$ & $6(24 \%)$ & $9(30 \%)$ & $6(26 \%)$ & $3(43 \%)$ \\
\hline Cardiovascular disease & & $5(9 \%)$ & $4(16 \%)$ & $1(3 \%)$ & $1(4 \%)$ & 0 \\
\hline Lung disease & & $12(22 \%)$ & $6(24 \%)$ & $6(20 \%)$ & $3(13 \%)$ & $3(43 \%)$ \\
\hline Immunosuppression & & $7(13 \%)$ & $5(20 \%)$ & $2(7 \%)$ & $1(4 \%)$ & $1(14 \%)$ \\
\hline \multicolumn{7}{|l|}{ Intercurrent events, n (\%) } \\
\hline Bact. superinfection & & $31(56 \%)$ & $5(20 \%)$ & $26(87 \%)^{e}$ & $20(87 \%)$ & $6(86 \%)$ \\
\hline ECMO & & $10(18 \%)$ & 0 & $10(33 \%)^{f}$ & $8(35 \%)$ & $2(29 \%)$ \\
\hline Thromboembolic complications & & $14(25 \%)$ & $1(4 \%)$ & $13(43 \%) g$ & $11(48 \%)$ & $2(29 \%)$ \\
\hline \multicolumn{7}{|l|}{ Treatment, $n(\%)$} \\
\hline Hydroxychloroquine & & $2(4 \%)$ & 0 & $2(7 \%)$ & $2(9 \%)$ & 0 \\
\hline Lopinavir/ritonavir & & $1(2 \%)$ & 0 & $1(3 \%)$ & $1(4 \%)$ & 0 \\
\hline Convalescent plasma & & $23(42 \%)$ & $5(20 \%)$ & $18(60 \%)^{h}$ & $12(52 \%)$ & $6(86 \%)$ \\
\hline Glucocorticoids & & $17(31 \%)$ & $5(20 \%)$ & $12(40 \%)$ & $9(39 \%)$ & $3(43 \%)$ \\
\hline Antibiotics & & $38(69 \%)$ & $9(36 \%)$ & $29(97 \%)^{i}$ & $23(100 \%)$ & $6(86 \%)$ \\
\hline \multicolumn{7}{|c|}{ 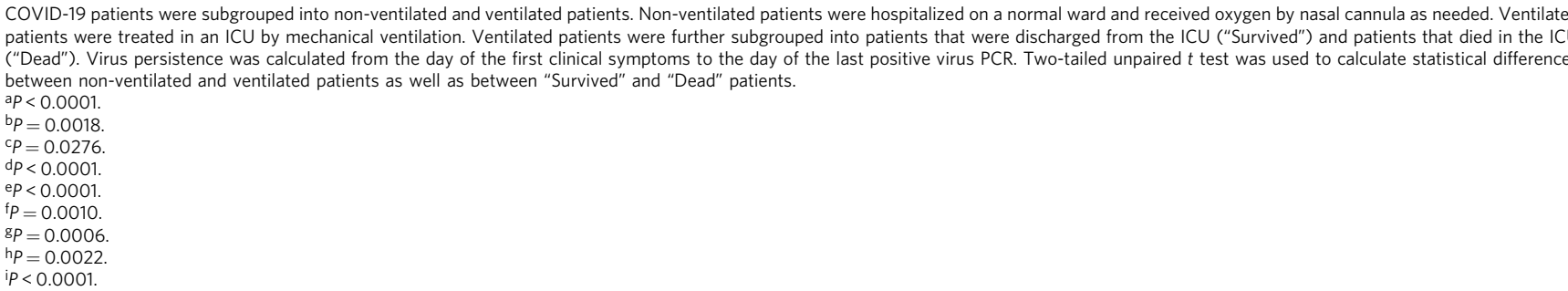 } \\
\hline
\end{tabular}

controls. Duration of T-cell hyporeactivity as defined by weak upregulation of CD123 and CD11b was significantly associated with prolonged viral replication in these patients (Supplementary Table 2). Absolute expression levels of surface markers on basophils, pDCs, monocytes, and neutrophils and representative FACS dot plots of unstimulated and anti-CD3-stimulated whole blood cells are shown in Supplementary Fig. 1 and Supplementary Fig. 3. Downregulation of CD131 on basophils was almost completely dependent on the release of IL-3 as shown with a blocking antibody against IL-3, while downregulation of CD131 on pDCs was only partially dependent on IL-3 (Supplementary Fig. 2a, b). Inhibition of IL-3 did not reduce the upregulation of CD123 on monocytes or CD11b on neutrophils (Supplementary Fig. 2c, d), most likely due to the high redundancy of T-cellderived signals with effects on monocytes and neutrophils (Supplementary Fig. 1). Stratification of ventilated COVID-19 patients according to their clinical outcome (survival vs. death) revealed that downregulation of CD131 on pDCs or basophils was low in both groups (Fig. 1b). In contrast, upregulation of CD123 on monocytes and CD11b on neutrophils, that integrate a larger set of signals from activated $\mathrm{T}$ cells, were almost absent in COVID-19 patients with fatal outcome, but still detectable in ventilated patients who recovered and were later discharged from the ICU (Fig. 1d, f). In healthy males and non-ventilated male COVID-19 patients, T-cell activation resulted in a significantly stronger upregulation of CD123 on monocytes than in females, suggesting a stronger T-cell-monocyte connection in males (Supplementary Fig. 4c). In ventilated COVID-19 patients, the impairment of T-cell-induced CD123 upregulation on monocytes was very pronounced in both genders (Supplementary Fig. 4c). Basophils, pDCs, and neutrophils responded equally to T-cell activation in males and females (Supplementary Fig. 4a, b, d).

To exclude that downstream responder cells like basophils, monocytes, and neutrophils are anergic to cytokine stimulation in COVID-19 patients, we incubated whole-blood samples of 10 healthy controls, 13 non-ventilated, and 15 ventilated patients with anti-CD3 or just with recombinant IL-3 and GM-CSF for 24 h (Supplementary Fig. 5). While the response of basophils, monocytes, and neutrophils to T-cell activation with anti-CD3 was very low in COVID-19 patients, their response to recombinant IL-3 and GM-CSF was fully preserved in a celltype-specific manner (preferential response of basophils to IL-3, of monocytes to IL-3 and GM-CSF, and of neutrophils to GMCSF).

CD4 + and CD8 + T-cell counts in whole-blood samples were similar in ventilated patients, non-ventilated patients, and healthy controls, arguing against the hypothesis that only differences in T-cell numbers account for the differences observed in whole blood after T-cell activation (Fig. 2a, b). Only in ventilated patients with fatal outcome CD $4+$ and $\mathrm{CD} 8+\mathrm{T}$-cell counts were reduced.

We also measured selected T-cell-derived cytokines by ELISA in the supernatant of T-cell-activated whole blood (Fig. 2c-f). We found no significant anti-CD3-induced release of IL-3, GM-CSF, and IFN-gamma in ventilated COVID-19 patients, but a considerable release in non-ventilated patients and healthy 
a

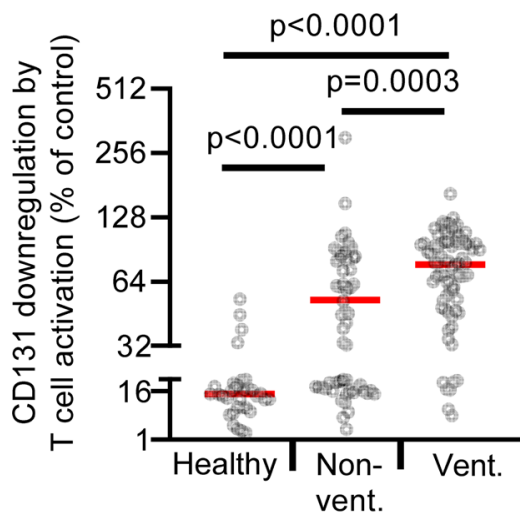

$\mathrm{C}$

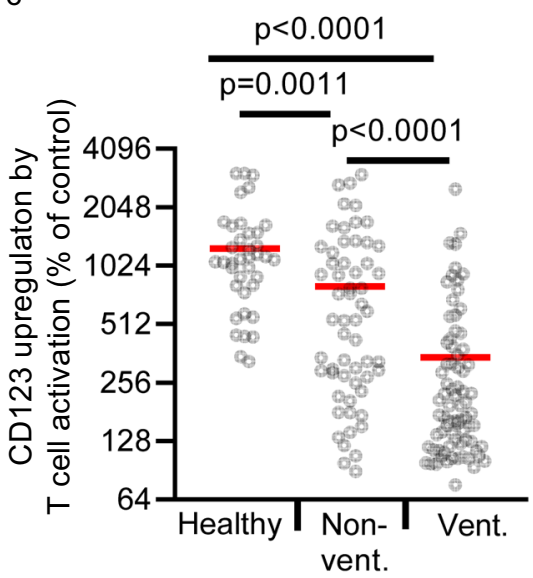

e

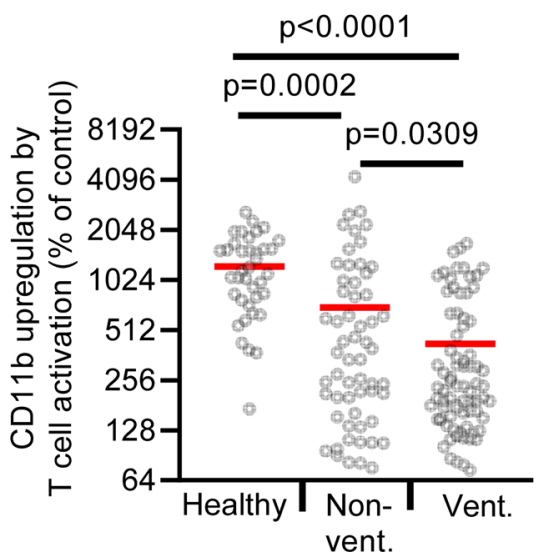

b

Basophils

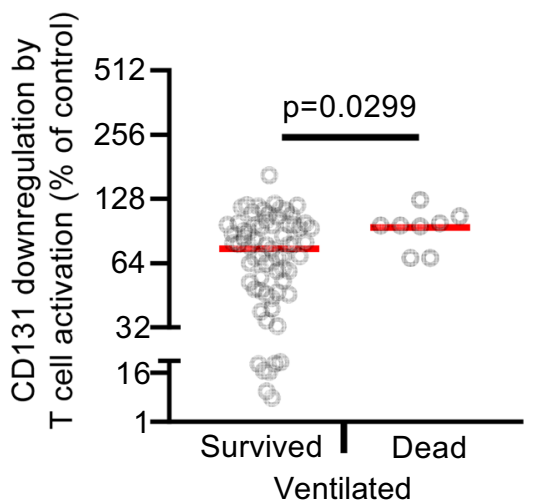

d

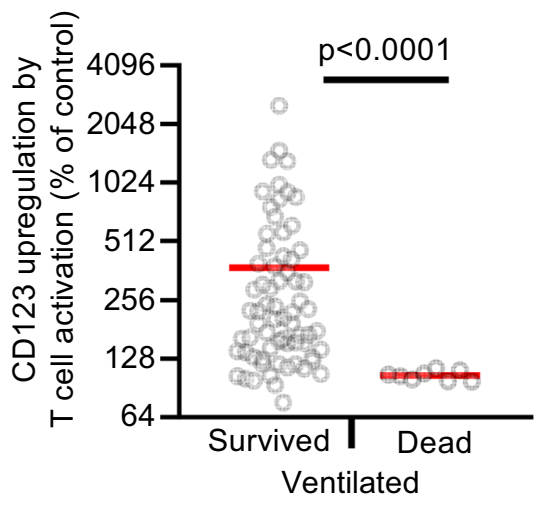

f

Neutrophils

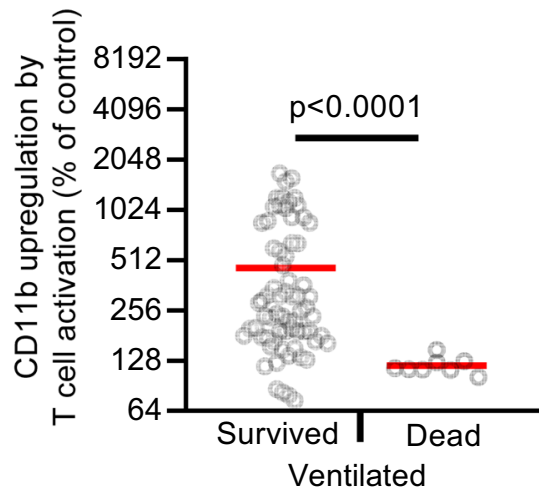

Fig. 1 T-cell reactivity in COVID-19 patients and healthy controls. a-f Whole-blood samples from 38 healthy controls (healthy; $n=38$ biologically independent samples), 33 non-ventilated (non-vent.; $n=58$ biologically independent samples), and 21 mechanically ventilated (vent.; $n=77$ biologically independent samples) COVID-19 patients were cultured with or without immobilized anti-CD3 for $24 \mathrm{~h}$. Ventilated patients were stratified into "survived" (17 patients, $n=69$ biologically independent samples) and "dead" (4 patients, $n=8$ biologically independent samples). Expression of indicated surface markers was quantified by flow cytometry on basophils (a, b), CD14 + monocytes (c, d), and neutrophils (e, f). Values depict the ratio of surface marker expression with anti-CD3 to surface marker expression without anti-CD3. Each sample is represented by one dot, and the mean is marked in red. One-way ANOVA with Bonferroni multiple comparison test was used for $\mathbf{a}, \mathbf{c}$, and $\mathbf{e}$. Two-tailed unpaired $t$ test with Welch's correction was used for $\mathbf{b}, \mathbf{d}$, and $\mathbf{f}$. Source data are provided as a Source Data file.

controls. These data are largely consistent with the above described downstream effects of T-cell activation on basophils, pDCs, monocytes, and neutrophils. However, the cytokine levels are more variable and show no significant differences between nonventilated patients and healthy controls. Thus, downstream cellular effects of T-cell activation are more sensitive than measurement of single cytokines by ELISA. Although some COVID-19 patients showed a high release of IL-10 in the supernatant of T-cellactivated whole blood, the difference between healthy controls and patients was not significant. The almost complete inhibition of 
a

$\mathrm{CD}^{+} \mathrm{T}$ cells

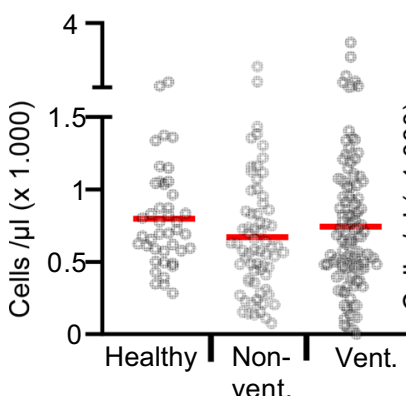

CD4 ${ }^{+} \mathrm{T}$ cells

b

$\mathrm{CD}^{+} \mathrm{T}$ cells

CD8 ${ }^{+} \mathrm{T}$ cells
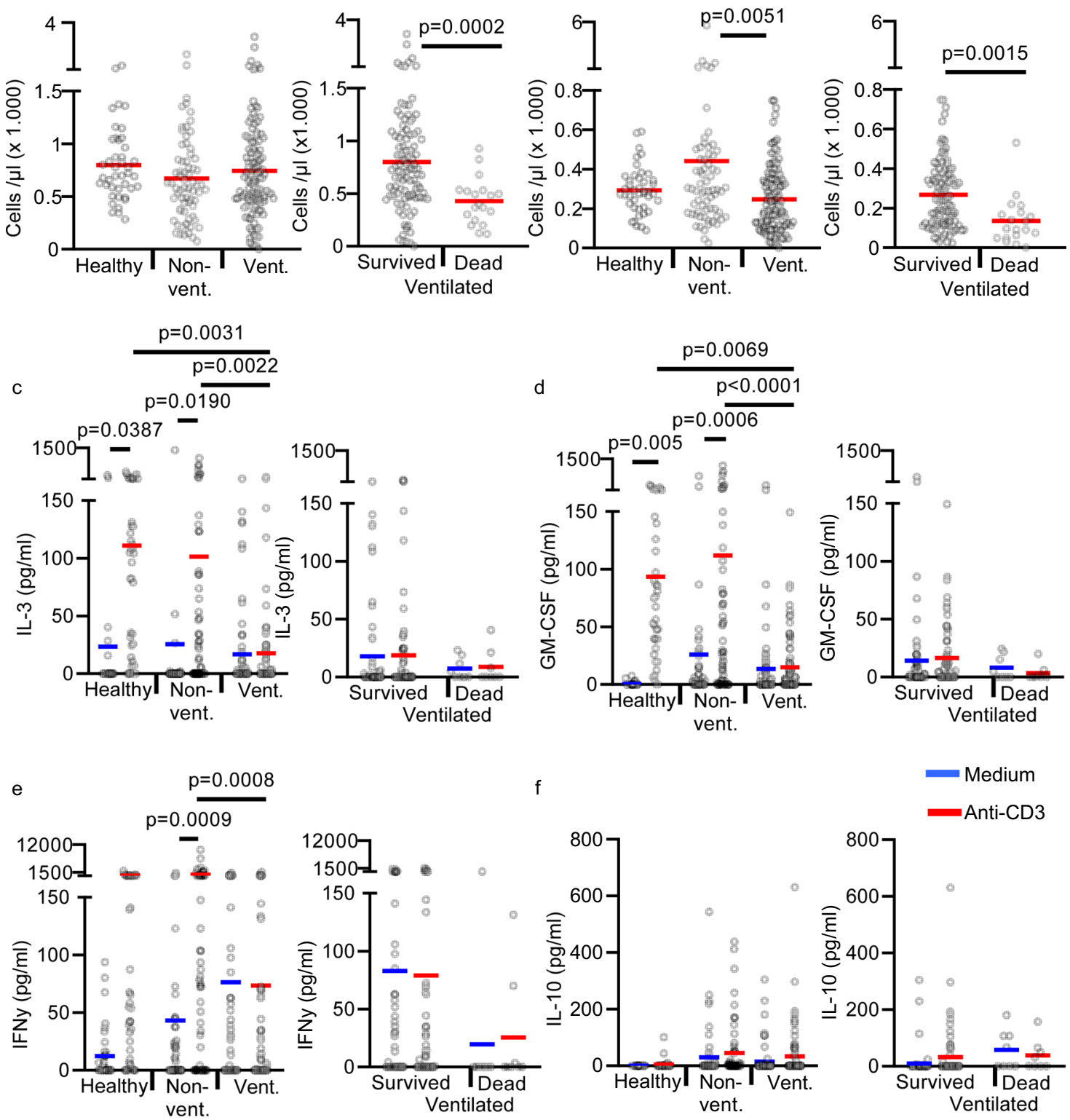

Fig. 2 T-cell counts and cytokine release in anti-CD3 activated whole-blood samples. a, b Absolute CD4 + and CD8 + T-cell counts in non-cultured fresh blood of 42 healthy controls (healthy; $n=42$ biologically independent samples), 39 non-ventilated COVID-19 patients (non-vent.; $n=68$ biologically independent samples) and 30 ventilated COVID-19 patients (vent.; $n=120$ biologically independent samples). Ventilated patients were stratified into "survived" (23 patients, $n=101$ biologically independent samples) and "dead" (7 patients, $n=19$ biologically independent samples). Each sample is represented by one dot, and the mean is marked in red. c-e Whole blood from 38 healthy controls (healthy; $n=38$ biologically independent samples), 33 non-ventilated (non-vent.; $n=58$ biologically independent samples) and 21 mechanically ventilated (vent.; $n=77$ biologically independent samples) COVID-19 patients was cultured with or without immobilized anti-CD3 for $24 \mathrm{~h}$. Ventilated patients were stratified into "survived" (17 patients, $n=69$ biologically independent samples) and "dead" ( 4 patients, $n=8$ biologically independent samples). Concentrations of IL-3 (c), GM-CSF (d), IFN- $\gamma$ (e), and IL-10 (f) were measured in the culture supernatant by ELISA. Each sample is represented by one dot, and the mean is marked in blue (medium) or red (anti-CD3). One-way ANOVA with Bonferroni multiple comparison test was used. Two-tailed unpaired $t$ test was used for the analysis of "Survived" and "Dead". Source data are provided as a Source Data file.

cytokine release from activated $\mathrm{T}$ cells in ventilated COVID-19 patients strongly suggests that both CD4 + and CD8 $+\mathrm{T}$ cells are hyporeactive, as IL-3 and IFN-gamma are released from both, $\mathrm{CD} 4+$ and $\mathrm{CD} 8+\mathrm{T}$ cells.

In order to find out whether T-cell hyporeactivity in COVID-19 patients is T-cell intrinsic or extrinsic, we exposed washed whole blood from a healthy donor with plasma from 10 healthy donors, 13 non-ventilated, and 19 ventilated COVID-19 patients. Plasma from non-ventilated patients and even more ventilated patients markedly suppressed T-cell activation, as shown by a much lower downregulation of CD131 on basophils and a much lower upregulation of CD123 on monocytes and CD11b on neutrophils (Fig. 3). The suppressive effects of COVID-19 plasma could not be explained by immunosuppressive medication, as only $23 \%$ of the non-ventilated and $33 \%$ of the ventilated patients received steroids. No other immunosuppressive agents were given to any of the patients. In Fig. 3, the plasma samples of patients with steroids are marked in blue and show no increased T-cell suppression. 

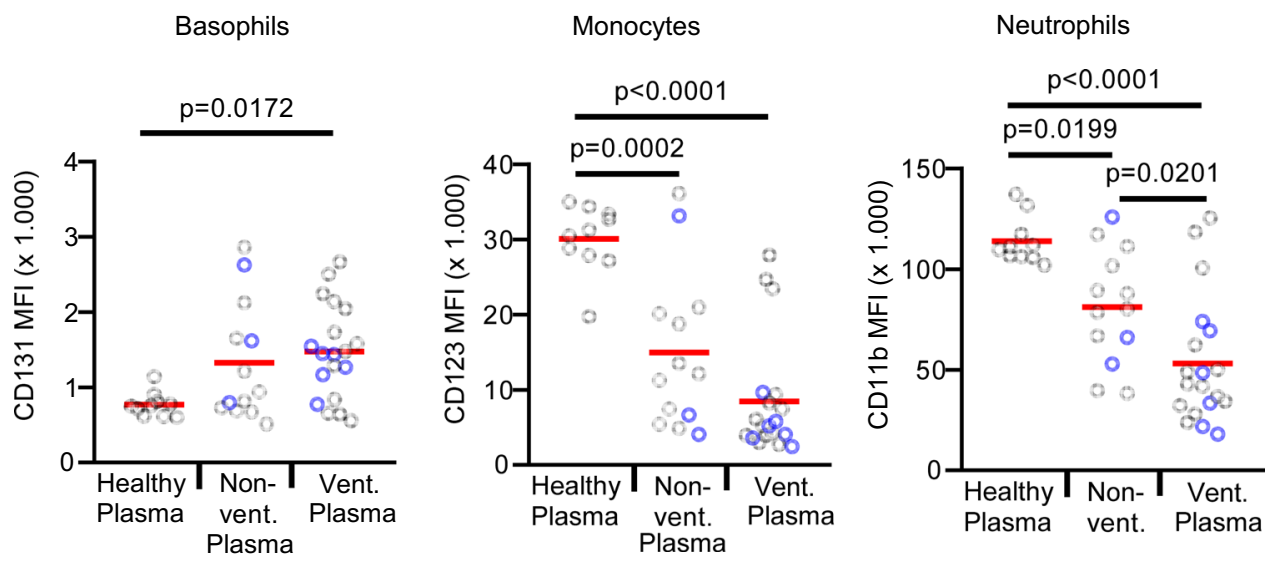

Fig. 3 Plasma from COVID-19 patients has pronounced T-cell suppressive activity. Whole blood from a healthy donor was washed twice with medium to remove the plasma. Plasma from 10 healthy controls (healthy plasma; $n=10$ biologically independent samples), 13 non-ventilated (non-vent. plasma; $n=$ 13 biologically independent samples), and 15 mechanically ventilated (vent. plasma; $n=19$ biologically independent samples) COVID-19 patients was added and samples were cultured with anti-CD3 for $24 \mathrm{~h}$. In all, $23 \%$ of the non-ventilated and $33 \%$ of the ventilated COVID-19 patients were treated with steroids (marked in blue). None of the patients was treated with other immunosuppressive agents. Expression of indicated surface markers was quantified by flow cytometry on basophils, CD14 + monocytes and neutrophils. The absolute expression values of indicated markers are shown as mean fluorescence intensity (MFI) on basophils, CD14 + monocytes, and neutrophils. Each sample is represented by one dot, and the mean is marked in red. One-way ANOVA with Bonferroni multiple comparison test was used. Source data are provided as a Source Data file.

We tested several approaches to overcome T-cell hyporeactivity in COVID-19 patients. The addition of recombinant IL-2 markedly improved $\mathrm{T}$-cell reactivity in non-ventilated patients but was less effective in ventilated patients (Supplementary Fig. 6). Blockade of IL-10 with a blocking antibody and addition of excess L-tryptophan to overcome potentially increased tryptophandegradation by indoleamine 2,3-dioxygenase (IDO) had no beneficial effects on T-cell reactivity (Supplementary Fig. 7).

PBMCs are not suitable to quantify polyclonal T-cell hyporeactivity in COVID-19 patients. We also used purified PBMCs to polyclonally activate the T cells with soluble anti-CD3 for $24 \mathrm{~h}$. Downstream effects of T-cell activation on pDCs and neutrophils could not be analyzed in PBMC samples, because neutrophils are absent and pDC numbers were too low. Basophils and monocytes responded only weakly to $\mathrm{T}$-cell-derived signals without significant differences between non-ventilated and ventilated patients (Supplementary Fig. 8a, b). Upregulation of CD25 on T cells (Supplementary Fig. $8 \mathrm{c}$, d) and release of T-cell-derived cytokines (Supplementary Fig. 8e) were clearly induced by antiCD3; however, only for GM-CSF a significant difference was detectable between non-ventilated and ventilated patients.

T-cell hyporeactivity is reversible in critically ill COVID-19 patients. In several COVID-19 patients, multiple blood samples were available over a prolonged period of time (Supplementary Fig. 9). Four cases of ventilated patients that could finally be weaned from mechanical ventilation and two cases of patients that died in the ICU are shown. Patients with fatal outcomes showed an almost complete T-cell hyporeactivity in multiple samples over up to 14 days. In contrast, T-cell hyporeactivity was less pronounced in patients with favorable outcomes. In general, their T-cell reactivity appeared to improve already before weaning and was improved after successful weaning. This shows on an individual basis that $\mathrm{T}$-cell hyporeactivity is reversible and correlates to disease severity.

Immunophenotyping of fresh blood samples in COVID-19 patients. We performed immunophenotyping of fresh blood samples of COVID-19 patients and healthy controls with a focus on innate cells like basophils, pDCs, monocytes, and neutrophils (Fig. 4). The gating strategy is shown in Supplementary Fig. 10. Basophil counts were markedly reduced in ventilated COVID-19 patients that subsequently died in the ICU (Fig. 4a). Consistent with published data, we also found decreased numbers of pDCs in ventilated COVID-19 patients (Fig. 4b).

Total neutrophil counts were higher in ventilated COVID-19 patients and also higher in ventilated patients with a fatal outcome (Fig. 4c). Expression of CD11b on neutrophils showed the same pattern as CD169 on monocyte. CD11b was significantly higher in non-ventilated COVID-19 patients and returned almost to baseline in ventilated and deceased patients (Fig. 4d).

CD16 + monocytes but not the classical CD14+ monocytes were decreased in non-ventilated and ventilated patients (Fig. $4 \mathrm{e}$, g). Both monocyte populations showed significantly increased expression of CD169 (Siglec-1) in non-ventilated patients compared to healthy controls (Fig. 4f, h). CD169 is one of the most prominent type I IFN-regulated genes on human monocytes $^{31}$. In ventilated COVID-19 patients, expression of CD169 returned almost to baseline, suggesting an impaired type I interferon production in critically ill COVID-19 patients. Reduced type I interferon responses were recently discovered by transcriptional profiling in critically ill COVID-19 patients ${ }^{32}$.

Design and assessment of a predictive score. The immunological characteristics of COVID-19 patients lead us to develop predictive scores for the survival of ventilated patients. We have incorporated three parameters that discriminated best between various groups of COVID-19 patients and calculated predictive values for the death of ventilated patients (Table 2). The first two parameters are CD123 upregulation on monocytes and CD11b-upregulation on neutrophils induced by anti-CD3 in whole blood with a cutoff of $130 \%$. Both parameters reflect the degree of T-cell hyporeactivity; however, they are not interchangeable, as monocytes and neutrophils sense different sets of T-cell-derived signals. The third parameter is the basophil count in the peripheral blood with a cutoff of $25 / \mu$ l. All three parameters should be easy to establish in different labs because the counting of basophils is routine and no standardized absolute expression levels are required for upregulation of CD123 and CD11b as only relative changes are calculated. By combination of all three 
a Basophils

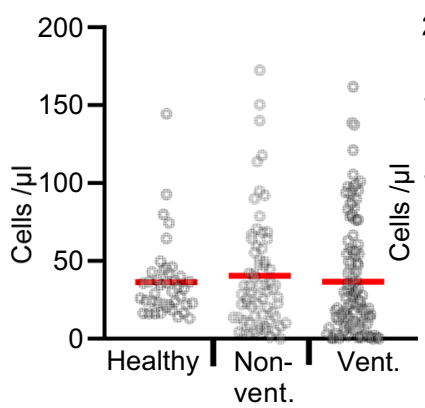

C Neutrophils

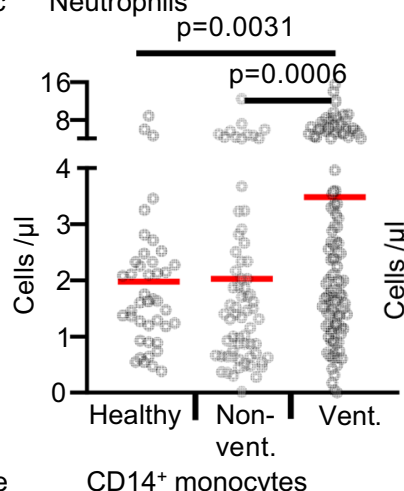

Basophils

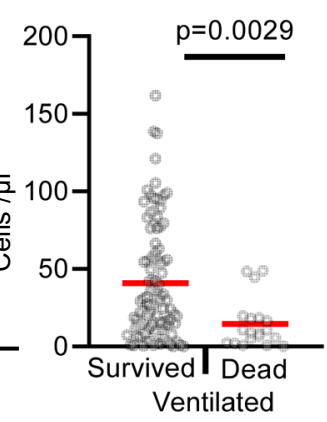

Neutrophils $p=0.0016$
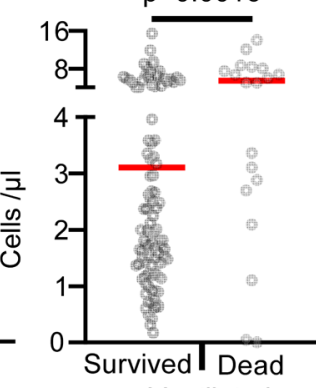

Ventilated CD $14^{+}$monocytes

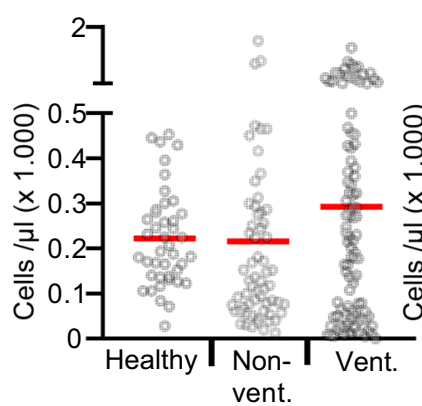

g CD16 monocytes

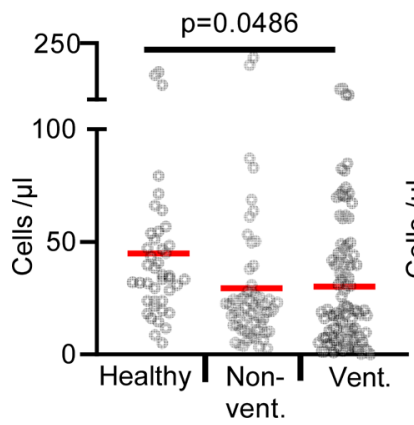

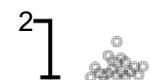

1886000

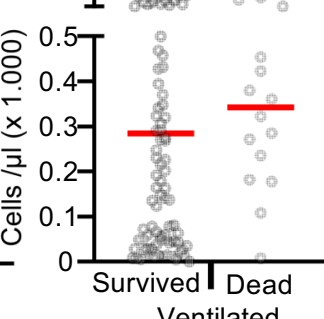

CD16 ${ }^{+}$monocytes

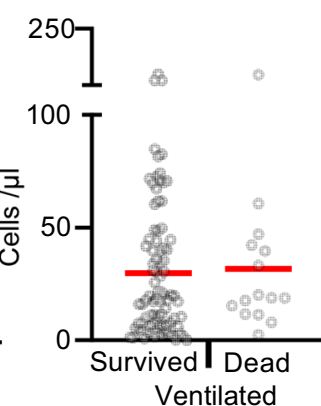

b pDCs

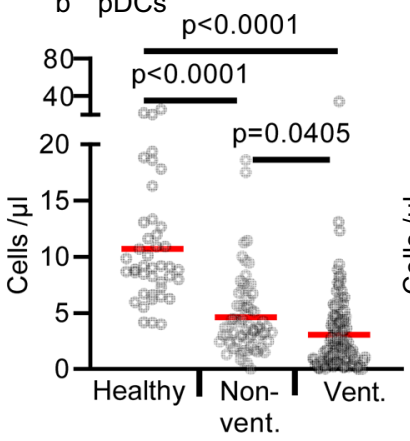

d Neutrophils

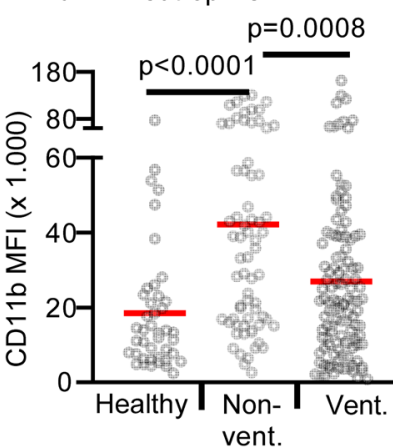

$\mathrm{CD} 14^{+}$monocytes

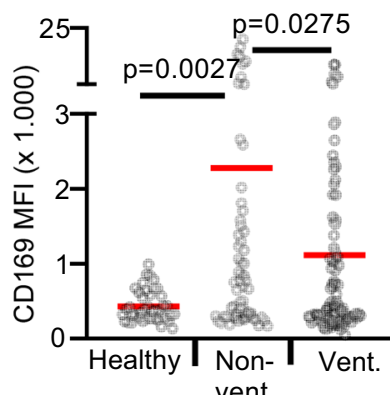

vent.

h $\mathrm{CD}_{16}{ }^{+}$monocytes

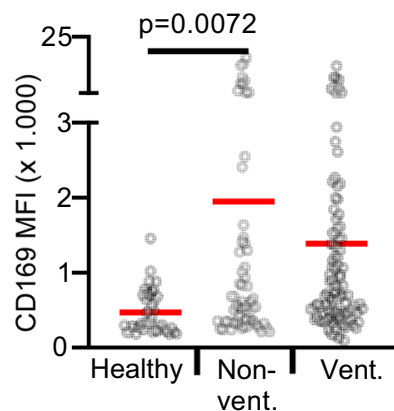

$\mathrm{pDCs}$

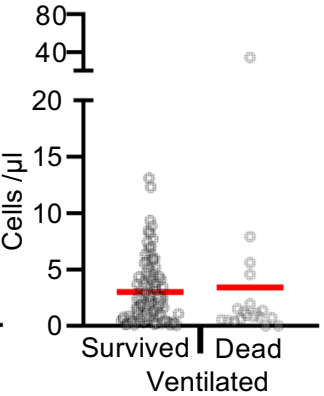

Neutrophils
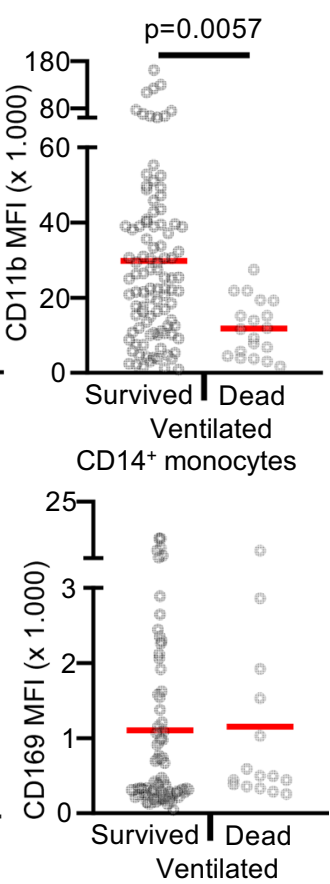

CD16 ${ }^{+}$monocytes

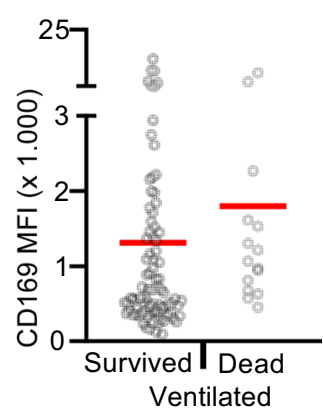

Fig. 4 Immunophenotyping of fresh peripheral blood cells in COVID-19 patients and healthy controls. a-d 42 healthy controls (healthy; $n=42$ biologically independent samples), 39 non-ventilated (non-vent.; $n=68$ biologically independent samples), and 30 mechanically ventilated (vent.; $n=120$ biologically independent samples) COVID-19 patients were analyzed. Ventilated patients were stratified into "survived" (23 patients, $n=101$ biologically independent samples) and "dead" (7 patients, $n=19$ biologically independent samples). a, b Absolute basophil and pDC counts. c, d Absolute neutrophils counts and their expression of CD11b given as mean fluorescence intensity (MFI). e-h 42 healthy controls (healthy; $n=42$ biologically independent samples), 38 non-ventilated (non-vent.; $n=62$ biologically independent samples), and 30 mechanically ventilated (vent.; $n=102$ biologically independent samples) COVID-19 patients were analyzed. Ventilated patients were stratified into "survived" (23 patients, $n=87$ biologically independent samples) and "dead" (7 patients, $n=15$ biologically independent samples). The number of samples is lower than in a-d because the antibodies for this panel were temporarily not available by suppliers. e, f Absolute CD14 + + CD16- monocyte counts and their surface expression of CD169. $\mathbf{g}, \mathbf{h}$ Absolute CD16 + monocyte counts and their surface expression of CD169. Each sample is represented by one dot, and the mean is marked in red. One-way ANOVA with Bonferroni multiple comparison test was used for the analysis of "healthy", "non-vent", and "vent". Two-tailed unpaired $t$ test was used for the analysis of "survived" and "dead". Source data are provided as a Source Data file. 
Table 2 Predictive score for death in ventilated COVID-19 patients.

\author{
Stimulated blood:
}

\begin{tabular}{|c|c|c|c|c|c|c|}
\hline 77 complete datasets of ventilated patients & $\begin{array}{l}\text { Right positive (of } \\
8 \text { dead) }\end{array}$ & $\begin{array}{l}\text { False positive (of } \\
69 \text { survived) }\end{array}$ & Sens. & Spe. & NPV & PPV \\
\hline $\begin{array}{l}\text { Weak upregulation of CD123 on Monos + weak upregulation of } \\
\text { CD11b on Neutros }\end{array}$ & 7 & 3 & $88 \%$ & $96 \%$ & $97 \%$ & $83 \%$ \\
\hline Weak upregulation of CD123 on Monos + low Baso count & 7 & 6 & $88 \%$ & $92 \%$ & $97 \%$ & $71 \%$ \\
\hline Weak upregulaiton of CD11b on Neutros & 7 & 7 & $88 \%$ & $90 \%$ & $97 \%$ & $67 \%$ \\
\hline
\end{tabular}

Three parameters were used individually or in combination to predict fatal outcome in ventilated COVID-19 patients. Absolute basophil counts in fresh peripheral blood. Basophil counts $<25 / \mu$ l defined "Low Baso count". Upregulation of CD123 on CD14 + monocytes and CD11b on neutrophils defined as the ratio of surface marker expression with anti-CD3 to surface marker expression without antiCD3. Upregulation of $<130 \%$ defined "Weak upregulation". In the combined scores, a logical AND combination was used that required all parameters to be fulfilled. For all three parameters, 77 datasets from 21 ventilated COVID-19 patients ( $n=77$ biologically independent samples) were available. In all, 17 ventilated patients were discharged from the ICU (survived) ( $n=69$ biologically independent samples) and 4 patients died on the ICU ( $n=8$ biologically independent samples). The number of right and false positive samples, the test sensitivity (Sens.), specificity (Spe.), negative (NPV), and positive predictive value (PPV) for predicting death are shown.

parameters, a positive predictive value of $100 \%$ and a negative predictive value of $94 \%$ were reached for fatal outcomes of patients on mechanical ventilation.

\section{Discussion}

We have shown that low polyclonal T-cell reactivity is a hallmark of COVID-19 patients and strongly correlated with disease severity. T-cell reactivity was much lower in ventilated than in non-ventilated hospitalized patients, which was again somewhat lower than in healthy controls. T-cell hyporeactivity was only detectable in assays with whole blood but not with purified PBMC and could be attributed to suppressive components present in the plasma of COVID-19 patients. Incubation of whole blood from a healthy donor with plasma samples from ventilated COVID-19 patients resulted in a pronounced polyclonal T-cell hyporeactivity. Neither immunosuppressive drugs potentially present in the plasma of COVID-19 patients nor IL-10 or degradation of Ltryptophan could explain the inhibitory effects of COVID-19 plasma. With PBMCs and classical methods to study T-cell activation conflicting data have been published showing unaltered, increased, or decreased polyclonal T-cell responses in critically ill COVID-19 patients $20,21,23-26$. Recently, impaired SARSCov-2-specific CD4 + and CD8 + T-cell responses were found to be associated with older age and more severe outcome, while humoral immune responses were not predictive ${ }^{27,28}$. Our data are in line with these findings and additionally suggest that critically ill COVID-19 have a more general T-cell suppression that could also impair the development of SARS-CoV-2-specific T-cell responses. Many critically ill COVID-19 patients suffer from fungal infections, which may be caused by the general T-cell suppression in these patients ${ }^{33,34}$.

Measurement of T-cell activation by downstream effects on responder cells was much more sensitive and suitable to detect impairment of T-cell activation and differences between groups of patients than measurement of T-cell-derived cytokines in the supernatant. The responder cells integrate a large set of signals from activated $\mathrm{T}$ cells in a very sensitive and cell-type-specific manner.

Our data indicate that low $\mathrm{T}$-cell reactivity rather than anergy of downstream responder cells accounts for the low response of basophils, pDCs, monocytes, and neutrophils, because relevant Tcell-derived cytokines like IL-3, GM-CSF, and IFN-gamma were reduced in a similar manner and neutralization of IL-3 markedly blocked the downstream effects on basophils. In addition, responder cells in patient samples were fully responsive towards stimulation with recombinant IL-3 and GM-CSF, further arguing against the energy of responder cells.

The severe T-cell hyporeactivity in critically ill COVID-19 patients does not seem to be a mere consequence of being critically ill, because $\mathrm{T}$-cell hyporeactivity was already seen in nonventilated COVID-19 patients. The appearance of T-cell hyporeactivity could be a counter-regulatory mechanism to the hyperinflammation present in COVID-19 patients or a strategy of the virus to interfere with specific host immune responses and may explain the prolonged viral replication seen in the group of ventilated patients. Prolonged viral replication in turn may result in more tissue destruction, more activation of innate immune cells by viral components and more inflammation. The pronounced T-cell hyporeactivity in critically ill patients with hyperinflammation strongly argues against a major direct role of $\mathrm{T}$ cells for hyperinflammation. Immunosuppressive approaches targeting $\mathrm{T}$ cells are unlikely to improve hyperinflammation, while strategies to overcome the pronounced T-cell hyporeactivity in critically ill COVID-19 patients could be a promising therapeutic approach to improve virus clearance without aggravating the hyperinflammation.

Immunophenotyping revealed low counts of basophils and pDCs and a low expression of CD11b on neutrophils in severely ill COVID-19 patients. It is known that IL-3 is the major cytokine to increase basophil counts ${ }^{30}$. IL-3 and/or GM-CSF are also important survival factors for basophils and $\mathrm{pDCs}^{35,36}$. Thus the lower counts of pDCs and basophils in critically ill patients may reflect not only their increased recruitment into tissues but also reduced survival and production of cells due to diminished secretion of IL-3 and GM-CSF by anergic T cells. Compared to healthy controls, basal CD11b expression on neutrophils is higher in non-ventilated and somewhat higher in ventilated patients. This indicates that COVID-19 leads to an activation of neutrophils. The pronounced T-cell hyporeactivity in ventilated patients may explain why basal CD11b expression is significantly lower in ventilated compared to non-ventilated patients and again lower in patients with a fatal outcome.

Gender-specific analysis showed that the T-cell-induced upregulation of CD123 on monocytes was significantly higher in healthy males and non-ventilated male patients than in the same groups of females. As IL-3 contributes to the activation of monocytes ${ }^{37}$, the stronger T-cell-monocyte link in males may explain the recently described higher plasma levels of IL-8 and IL18 in male COVID-19 patients $^{38}$. 
We have developed a score based on downstream effects of Tcell activation on monocytes and neutrophils, and absolute basophil counts to predict fatal outcome in ventilated COVID-19 patients. We would like to point out, that the score must be confirmed in additional cohorts of COVID-19 patients. The scores may be clinically useful to identify patients that may benefit from strategies to overcome T-cell hyporeactivity.

Collectively our data suggest that approaches to overcome T-cell hyporeactivity combined with concepts to specifically suppress hyperinflammation induced by innate immune cells, particularly monocytes/macrophages could be promising immunotherapy for critically ill COVID-19 patients.

\section{Methods}

Study subjects and sampling. A total of 55 adult patients diagnosed with COVID-19 after testing positive for SARS-CoV-2 RNA by qPCR and 42 adult healthy volunteers without any clinical signs of COVID-19 infection were enrolled from March to July 2020 at the University Hospital Regensburg. This study was approved by the Research Ethics Committee from the University Hospital Regensburg (Study and Approval Number: 20-1785-101). The study was performed in accordance with all relevant ethical regulations for work with human participants. Informed consent and consent to publish were obtained from all enrolled patients (or their legal representatives) and healthy volunteers.

The cohort of COVID-19 patients was stratified into non-ventilated $(n=25)$ and mechanically ventilated $(n=30)$ patients. Ventilated patients were further subgrouped according to their outcome (discharged from the ICU = "survived", or death on the ICU = "dead") (Table 1). In most patients, several consecutive blood samples were available resulting in a total of 188 samples. In total, 14 patients were first sampled on mechanical ventilation and also after discharge from the ICU. Samples of these patients were assigned into the subgroup "ventilated" or "nonventilated" according to the ventilation status at the time of sampling (Supplementary Table 1). None of the patients received prednisolone equivalents $\geq 40 \mathrm{mg} /$ day for treatment of COVID-19.

Fresh whole-blood samples for immediate immunophenotyping by flow cytometry with at least one antibody panel were available from all participants. Sufficient amounts of fresh blood to also perform whole-blood stimulation were available from 38 healthy controls (38 samples), 33 non-ventilated COVID-19 patients (58 samples), and 21 mechanically ventilated COVID-19 patients (77 samples). For subgroup analysis the mechanically ventilated patients were again stratified according to their outcome into the groups "survived" (17 patients, 69 samples) and "dead" (4 patients, 8 samples). Peripheral blood mononuclear cells (PBMCs) were prepared from 25 non-ventilated patients (36 samples) and 16 ventilated COVID-19 patients (42 samples). For subgroup analysis, the mechanically ventilated patients were again stratified according to their outcome into the groups "survived" ( $n=14,39$ samples) and "dead" ( $n=2,3$ samples). The exact numbers of patients and samples for each readout are provided in the figure legends, as for some readouts, not enough material was available.

Cell culture media, cytokines, and stimulants. For PBMC cultures, we used RPMI 1640 medium (Gibco, Karlsruhe, Germany) containing 1\% penicillin/streptomycin (Gibco, Karlsruhe, Germany) and 1\% L-glutamine (Gibco, Karlsruhe, Germany). For whole-blood stimulations, we used pure RPMI 1640 medium (Gibco, Karlsruhe, Germany). Anti-CD3 (clone OKT3, eBiosciences, San Diego, CA, Cat. \# 16-0037-85) was used at $5 \mu \mathrm{g} / \mathrm{ml}$. The blocking anti-IL-3 antibody (clone P8C11) was generated in our $\mathrm{lab}$ and used at a concentration of $10 \mu \mathrm{g} / \mathrm{ml}$. The blocking anti-IL-10 antibody (clone JES3-19F1, BioLegend, San Diego, USA,, Cat. \# 506813) was used at $20 \mu \mathrm{g} / \mathrm{ml}$. IL-3 (BioLegend) and GM-CSF, IL-2, IL-4, IL-5, IL-6, IL-15, IFN- $\gamma$, IFN- $\alpha$, TNF- $\alpha$ (Peprotech, Cranbury, USA) were used at $20 \mathrm{ng} / \mathrm{ml}$. L-tryptophan (Sigma-Aldrich, Darmstadt, Germany) was used at $100 \mu \mathrm{g} / \mathrm{ml}$.

Whole-blood stimulation. For T-cell activation, assays tubes (BD Bioscience) were precoated with $300 \mu \mathrm{l}$ anti-CD3 antibodies $\left(5 \mu \mathrm{g} / \mathrm{ml}\right.$ in PBS) at $37^{\circ} \mathrm{C}$ for $4 \mathrm{~h}$ and washed twice with PBS. In all, $100 \mu$ l of heparinized blood were diluted with $200 \mu \mathrm{l}$ RPMI 1640 medium and added to the precoated or to uncoated tubes and incubated at $37^{\circ} \mathrm{C}$ in $5 \% \mathrm{CO}_{2}$ for $24 \mathrm{~h}$. Where indicated a blocking anti-IL-3 antibody $(10 \mu \mathrm{g} /$ $\mathrm{ml})$, a blocking anti-IL-10 antibody $(20 \mu \mathrm{g} / \mathrm{ml}), \mathrm{IL}-2(20 \mathrm{ng} / \mathrm{ml}), \mathrm{GM}-\mathrm{CSF}(10 \mathrm{ng} /$ $\mathrm{ml})$, or IL-3 $(10 \mathrm{ng} / \mathrm{ml})$ were added to the samples. The supernatants were recovered and released cytokines analyzed by ELISA. Cells were analyzed by multi-parametric flow cytometry. For cytokine stimulation, $100 \mu \mathrm{l}$ whole blood was diluted with 200 $\mu$ RPMI 1640 medium and added to uncoated tubes (BD Bioscience) together with $20 \mathrm{ng} / \mathrm{ml}$ of cytokines (IL-2, IL-3, IL-4, IL-5, IL-6, IL-15, GM-CSF, IFN- $\gamma$, IFN- $\alpha$, and TNF- $\alpha$ ) at $37^{\circ} \mathrm{C}$ in $5 \% \mathrm{CO}_{2}$ for $24 \mathrm{~h}$. Cells were analyzed by flow cytometry.

Whole-blood stimulation with the transfer of plasma. Assay tubes (BD Bioscience) were precoated with $300 \mu \mathrm{l}$ anti-CD3 antibodies ( $5 \mu \mathrm{g} / \mathrm{ml}$ in PBS) at 37 ${ }^{\circ} \mathrm{C}$ for $4 \mathrm{~h}$ and washed twice with PBS. Heparinized whole blood from a healthy donor was washed twice with medium to remove the plasma. In each precoated or uncoated assay tubes, $100 \mu \mathrm{l}$ of washed whole blood, $150 \mu \mathrm{l}$ of the medium, and 50 $\mu \mathrm{l}$ of heparinized plasma from healthy controls, non-ventilated and ventilated COVID-19 patients was added and samples were cultured at $37^{\circ} \mathrm{C}$ in $5 \% \mathrm{CO}_{2}$ for $24 \mathrm{~h}$. Cells were analyzed by multi-parametric flow cytometry.

Preparation and culture of PBMCs. Peripheral blood mononuclear cells (PBMCs) were prepared by Ficoll-Paque density gradient centrifugation from heparinanticoagulated fresh blood samples. For cryopreservation, PBMCs were resuspended in fetal calf serum (FCS) with $10 \%$ dimethylsulfoxide (DMSO) at a concentration of $1-2 \times$ $10^{6} \mathrm{cells} / \mathrm{ml}$, frozen at $-80^{\circ} \mathrm{C}$ for $2-3$ days and then transferred into liquid nitrogen. The cells were thawed in a $37^{\circ} \mathrm{C}$ water bath and washed three times with medium. The viability was controlled by Trypan-blue staining and was $90-95 \%$. PBMCs (500.000/ well) were cultured in $300 \mu \mathrm{l}$ medium/well with or without anti-CD3 (clone: OKT3) 5 $\mu \mathrm{g} / \mathrm{ml}$ for $24 \mathrm{~h}$ by $37^{\circ} \mathrm{C}$ and $5 \% \mathrm{CO}_{2}$. Thereafter supernatants were recovered and analyzed by ELISA. Cells were analyzed by flow cytometry.

Flow cytometry. Anticoagulated fresh blood samples $(100 \mu \mathrm{l})$ or cells after wholeblood stimulations were incubated with various panels of the following directly labeled monoclonal antibodies for $20 \mathrm{~min}$ by $4{ }^{\circ} \mathrm{C}$ : anti-hCD3 APC-Cy7 (clone: SK7, BioLegend, Cat. \# 344818, dilution 1:100), anti-hCD4 V500 (clone RPA-T4, BD Bioscience, Cat. \# 560768, dilution 1:100), anti-hCD8 APC-eFluor 780 (clone: RPA-T8, Invitrogen, Darmstadt, Germany, Cat. \# 47-0088-42, dilution 1:40), antihCD8 PE-Cy7 (clone: SK1, BioLegend, Cat. \# 344712, dilution 1:100), anti-hCD11b PE-Cy7 (clone: M1/70, BioLegend, San Diego, CA, Cat. \# 101216, dilution 1:100), anti-hCD14 V500 (clone: MФP9, BD Bioscience, Cat. \# 562693, dilution 1:100), anti-hCD16 Pacific Blue (clone: 3G8, BioLegend, Cat. \# 302032, dilution 1:100), anti-hCD19 Pacific Blue (clone: HIB19, BioLegend, Cat. \# 302232, dilution 1:100), anti-hCD25 APC (clone: BC96, BioLegend, Cat. \# 302610, dilution 1:40), antihCD116 FITC (clone: 4H1, BioLegend, Cat. \# 305906, dilution 1:40), anti-hCD131 PE (clone: 1C1, eBioscience, Cat. \# 12-1319-42, dilution 1:100), anti-hCD123 PECy5 (clone: 9F5, BD Bioscience, Cat. \# 551065, dilution 1:20), anti-hCD169 PE (clone: 7-239, Miltenyi Biotec, Bergisch Gladbach, Germany, Cat. \# 130-098-654 dilution 1:22), anti-hCD304 APC (clone: 12C2, BioLegend, Cat. \# 354506, dilution 1:40), anti-hHLA-DR II APC (clone: G-46-6, BD Bioscience, Cat. \# 559866, dilution 1:100) and FITC (clone: G-46-6, BD Bioscience, Cat. \# 555811, dilution 1:100) Subsequently, samples were treated with FACS Lysing Solution (BD Bioscience) for 10 min, washed with $0.9 \% \mathrm{NaCl}$, centrifuged, resuspended in $0.9 \% \mathrm{NaCl}$ together with FACS counting beads (Invitrogen, Darmstadt, Germany) and acquired with a BD FACSCanto II flow cytometer and analyzed with FACSDIVA 8 software (BD Biosciences). For analysis of cultured PBMCs, no erythrocyte lysis was performed. The gating strategy for key cell populations is shown in Supplementary Fig. 10.

Enzyme-linked immunosorbent assay (ELISA). Concentrations of GM-CSF, IFNgamma, and IL-10 in cell culture supernatants were measured by ELISA, according to the manufacturer's protocol (DuoSet Elisa, R\&D Systems, Abington, UK). For measurement of IL-3, ELISA plates (NUNC Maxisorb, Thermofisher Scientific, Waltham, USA) were coated with a capture anti-IL-3 antibody (Clone P8C11; $5 \mu \mathrm{g} / \mathrm{ml}$ in PBS) in $100 \mu \mathrm{l} /$ well at room temperature (RT) overnight. Plates were washed three times with PBS/0.05\% Tween-20, blocked with PBS containing $1 \%$ bovine serum albumin (BSA) at RT for $1 \mathrm{~h}$, and washed again with PBS. Samples were preincubated with $100 \mu \mathrm{g} / \mathrm{ml}$ mouse IgG1, kappa isotype control antibody (MOPC21, BioXCell, Lebanon, USA) for $1 \mathrm{~h}$ by RT and added to the plates for $2 \mathrm{~h}$ by RT ( $100 \mu \mathrm{l} /$ well, diluted in PBS/1\% BSA). Recombinant human IL-3 (BioLegend) was diluted from 7.8 to $500 \mathrm{pg} / \mathrm{ml}$ in PBS/1\% BSA and served as standard. After washing, plates were incubated with $400 \mathrm{ng} / \mathrm{ml} \mathrm{HRP-}$ labeled detection anti-IL-3 antibody (Clone 13) (100 $\mu \mathrm{l} /$ well) for $1.5 \mathrm{~h}$ at RT, and color reaction was performed with TMB Substrate Solution (BioLegend) according to the manufacturer's protocol.

\section{The predictive score for death in ventilated COVID-19 patients. Three para-} meters were used individually or in combination to predict fatal outcome in ventilated COVID-19 patients. Absolute basophil counts in fresh peripheral blood Basophil counts $<25 / \mu$ l defined "Low Baso count". Upregulation of CD123 on $\mathrm{CD} 14+$ monocytes and $\mathrm{CD} 11 \mathrm{~b}$ on neutrophils was calculated as the ratio of surface marker expression with anti-CD3 and surface marker expression without anti-CD3. Upregulation of $<130 \%$ defined "Weak upregulation". In the combined scores, a logical AND combination was used that required all parameters to be fulfilled. For all three parameters, 77 datasets from 21 ventilated COVID-19 patients were available. In total, 17 ventilated patients were discharged from the ICU (survived) (69 samples) and 4 patients died on the ICU ( 8 samples). The number of right and false-positive samples, the test sensitivity (Sens.), specificity (Spe.) were calculated. The negative (NPV) and positive predictive value (PPV) for predicting death were calculated using a prevalence of death in ventilated COVID19 patients of $19 \%$ (4 dead patients/21 ventilated patients).

Statistics and reproducibility. Statistical analyses were performed using the GraphPad Prism 8 Software. Statistical differences between more than two different cell stimulations or patient cohorts were calculated using one-way ANOVA with Bonferroni multiple comparisons, as indicated in the figures. For two-group comparisons, two- 
tailed unpaired $t$ test was used as indicated in the figures. In Supplementary Fig. 1, the experimental findings were successfully replicated twice. In all other cases, no replications were done, because only one sample per patient per day was available.

Reporting summary. Further information on experimental design is available in the Nature Research Reporting Summary linked to this paper.

\section{Data availability}

The datasets generated during and/or analyzed during this study are available from the corresponding author on reasonable request. Source data are provided with this paper.

Received: 11 September 2020; Accepted: 21 April 2021;

Published online: 21 May 2021

\section{References}

1. Zhu, N. et al. A Novel coronavirus from patients with pneumonia in China, 2019. N. Engl. J. Med. 382, 727-733 (2020).

2. Huang, C. et al. Clinical features of patients infected with 2019 novel coronavirus in Wuhan, China. Lancet 395, 497-506 (2020).

3. Zheng, Z. et al. Risk factors of critical \& mortal COVID-19 cases: a systematic literature review and meta-analysis. J. Infect. 81, e16-e25 (2020).

4. Grasselli, G. et al. Risk factors associated with mortality among patients with COVID-19 in intensive care units in Lombardy, Italy. JAMA Intern. Med. (2020).

5. Petersen, A. et al. The role of visceral adiposity in the severity of COVID-19: highlights from a unicenter cross-sectional pilot study in Germany. Metabolism 110, 154317 (2020).

6. Zhou, F. et al. Clinical course and risk factors for mortality of adult inpatients with COVID-19 in Wuhan, China: a retrospective cohort study. Lancet 395, 1054-1062 (2020).

7. McFadyen, J. D., Stevens, H. \& Peter, K. The emerging threat of (micro) thrombosis in COVID-19 and its therapeutic implications. Circ. Res. 127, 571-587 (2020).

8. Channappanavar, R. et al. Dysregulated type I interferon and inflammatory monocyte-macrophage responses cause lethal pneumonia in SARS-CoVinfected mice. Cell Host Microbe 19, 181-193 (2016).

9. Merad, M. \& Martin, J. C. Pathological inflammation in patients with COVID19: a key role for monocytes and macrophages. Nat. Rev. Immunol. 20 , 355-362 (2020).

10. Martinez, F. O., Combes, T. W., Orsenigo, F. \& Gordon, S. Monocyte activation in systemic Covid-19 infection: assay and rationale. eBioMedicine 59, 102964 (2020).

11. Mosser, D. M. \& Edwards, J. P. Exploring the full spectrum of macrophage activation. Nat. Rev. Immunol. 8, 958-969 (2008).

12. Le Bert, N. et al. SARS-CoV-2-specific T cell immunity in cases of COVID-19 and SARS, and uninfected controls. Nature 584, 457-462 (2020).

13. Braun, J. et al. SARS-CoV-2-reactive $\mathrm{T}$ cells in healthy donors and patients with COVID-19. Nature https://doi.org/10.1038/s41586-020-2598-9 (2020).

14. Grifoni, A. et al. Targets of T cell responses to SARS-CoV-2 coronavirus in humans with COVID-19 disease and unexposed individuals. Cell 181, 1489-1501 e1415 (2020).

15. Ni, L. et al. Detection of SARS-CoV-2-specific humoral and cellular immunity in COVID-19 convalescent individuals. Immunity 52, 971-977 e973 (2020)

16. Shankar-Hari, M., Estcourt, L., Harvala, H., Roberts, D. \& Menon, D. K. Convalescent plasma to treat critically ill patients with COVID-19: framing the need for randomised clinical trials. Crit. Care 24, 449 (2020).

17. Piechotta, V. et al. Convalescent plasma or hyperimmune immunoglobulin for people with COVID-19: a living systematic review. Cochrane Database Syst. Rev. 7, CD013600 (2020)

18. Tomazini, B. M. et al. Effect of dexamethasone on days alive and ventilatorfree in patients with moderate or severe acute respiratory distress syndrome and COVID-19: the CoDEX randomized clinical trial. J. Am. Med. Assoc. https://doi.org/10.1001/jama.2020.17021 (2020).

19. Horby, P. et al. Dexamethasone in hospitalized patients with Covid-19preliminary report. N. Engl. J. Med. https://doi.org/10.1056/NEJMoa2021436 (2020).

20. Mazzoni, A. et al. Impaired immune cell cytotoxicity in severe COVID-19 is IL-6 dependent. J. Clin. Investig. 130, 4694-4703, https://doi.org/10.1172/ JCI138554 (2020).

21. Zhou, R. et al. Acute SARS-CoV-2 infection impairs dendritic cell and T cell responses. Immunity https://doi.org/10.1016/j.immuni.2020.07.026 (2020)

22. Mathew, D. et al. Deep immune profiling of COVID-19 patients reveals distinct immunotypes with therapeutic implications. Science 369, https://doi. org/10.1126/science.abc8511 (2020).
23. Wang, F. et al. The laboratory tests and host immunity of COVID-19 patients with different severity of illness. JCI Insight 5, https://doi.org/10.1172/jci. insight.137799 (2020)

24. De Biasi, S. et al. Marked T cell activation, senescence, exhaustion and skewing towards TH17 in patients with COVID-19 pneumonia. Nat. Commun. 11, 3434 (2020).

25. Chen, Z. \& John Wherry, E. T cell responses in patients with COVID-19. Nat Rev. Immunol. 20, 529-536 (2020).

26. Chen, G. et al. Clinical and immunological features of severe and moderate coronavirus disease 2019. J. Clin. Investig. 130, 2620-2629 (2020).

27. Rydyznski Moderbacher, C. et al. Antigen-specific adaptive immunity to SARS-CoV-2 in acute COVID-19 and associations with age and disease severity. Cell 183, 996-1012 e1019 (2020).

28. Tan, A. T. et al. Early induction of functional SARS-CoV-2-specific T cells associates with rapid viral clearance and mild disease in COVID-19 patients. Cell Rep. 34, 108728 (2021).

29. Ou, M. et al. Risk factors of severe cases with COVID-19: a meta-analysis. Epidemiol. Infect. 148, e175 (2020).

30. Dougan, M., Dranoff, G. \& Dougan, S. K. GM-CSF, IL-3, and IL-5 family of cytokines: regulators of inflammation. Immunity 50, 796-811 (2019).

31. Biesen, R. et al. Sialic acid-binding Ig-like lectin 1 expression in inflammatory and resident monocytes is a potential biomarker for monitoring disease activity and success of therapy in systemic lupus erythematosus. Arthritis Rheum. 58, 1136-1145 (2008)

32. Hadjadj, J. et al. Impaired type I interferon activity and inflammatory responses in severe COVID-19 patients. Science 369, 718-724 (2020).

33. Evert, K. et al. Autopsy findings after long-term treatment of COVID-19 patients with microbiological correlation. Virchows Arch. 1-12. https://doi. org/10.1007/s00428-020-03014-0 (2021).

34. Kuehn, B. M. Pulmonary fungal infections affect patients with COVID-19. J. Am. Med. Assoc. 324, 2248, https://doi.org/10.1001/jama.2020.22914 (2020).

35. Lepelletier, Y. et al. Toll-like receptor control of glucocorticoid-induced apoptosis in human plasmacytoid predendritic cells (pDCs). Blood 116, 3389-3397 (2010)

36. Didichenko, S. A., Spiegl, N., Brunner, T. \& Dahinden, C. A. IL-3 induces a Piml-dependent antiapoptotic pathway in primary human basophils. Blood 112, 3949-3958 (2008).

37. Weber, G. F. et al. Interleukin-3 amplifies acute inflammation and is a potential therapeutic target in sepsis. Science 347, 1260-1265 (2015).

38. Takahashi, T. et al. Sex differences in immune responses that underlie COVID-19 disease outcomes. Nature https://doi.org/10.1038/s41586-020-2700-3 (2020).

\section{Acknowledgements}

This work was supported by a grant from the Bavarian Ministry of Science and Arts.

\section{Author contributions}

M.M. conceived and supervised the study. K.R. and M.M. analyzed the data. T.S., S.C., J.G C.M., C.T., J.-N.S., F.W., S.Ba., and S.Bu. performed all experiments. M.V.M. provided patient data. M.L., D.L., B.G., F.Ha., F.Hi., and B.S. provided patient samples and clinical data. H.P. obtained ethical approval for the study, M.K. provided healthy control samples. E.O. and R.B. provided laboratory values and organized sample distribution. T.N., C.B., and A.G. organized biobanking of PBMCs. M.M. and K.R. drafted the manuscript.

\section{Funding}

Open Access funding enabled and organized by Projekt DEAL.

\section{Competing interests}

The authors declare no competing interests.

\section{Additional information}

Supplementary information The online version contains supplementary material available at https://doi.org/10.1038/s41467-021-23334-2.

Correspondence and requests for materials should be addressed to M.M.

Peer review information Nature Communications thanks Antonio Bertoletti and Grégory Verdeil for their contribution to the peer review of this work. Peer reviewer reports are available.

Reprints and permission information is available at http://www.nature.com/reprints

Publisher's note Springer Nature remains neutral with regard to jurisdictional claims in published maps and institutional affiliations. 
(c) (i) Open Access This article is licensed under a Creative Commons Attribution 4.0 International License, which permits use, sharing, adaptation, distribution and reproduction in any medium or format, as long as you give appropriate credit to the original author(s) and the source, provide a link to the Creative Commons license, and indicate if changes were made. The images or other third party material in this article are included in the article's Creative Commons license, unless indicated otherwise in a credit line to the material. If material is not included in the article's Creative Commons license and your intended use is not permitted by statutory regulation or exceeds the permitted use, you will need to obtain permission directly from the copyright holder. To view a copy of this license, visit http://creativecommons.org/ licenses/by/4.0/.

(C) The Author(s) 2021 